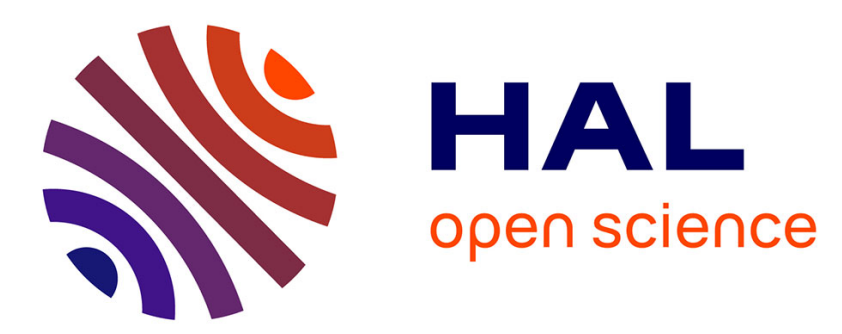

\title{
Complementary speckle patterns: deterministic interchange of intrincic vortices and maxima throuhg scattering media
}

\author{
Jérôme Gateau, Hervé Rigneault, Marc Guillon
}

\section{- To cite this version:}

Jérôme Gateau, Hervé Rigneault, Marc Guillon. Complementary speckle patterns: deterministic interchange of intrincic vortices and maxima throuhg scattering media. Physical Review Letters, 2017, 118 (4), pp. 043903. 10.1103/PhysRevLett.118.043903 . hal-01450021

\section{HAL Id: hal-01450021 \\ https://hal.science/hal-01450021}

Submitted on 18 Apr 2018

HAL is a multi-disciplinary open access archive for the deposit and dissemination of scientific research documents, whether they are published or not. The documents may come from teaching and research institutions in France or abroad, or from public or private research centers.
L'archive ouverte pluridisciplinaire HAL, est destinée au dépôt et à la diffusion de documents scientifiques de niveau recherche, publiés ou non, émanant des établissements d'enseignement et de recherche français ou étrangers, des laboratoires publics ou privés. 


\title{
Complementary Speckle Patterns: Deterministic Interchange of Intrinsic Vortices and Maxima through Scattering Media
}

\author{
Jérôme Gateau, ${ }^{1}$ Hervé Rigneault, ${ }^{2}$ and Marc Guillon ${ }^{3, *}$ \\ ${ }^{1}$ Holographic Microscopy Group, Neurophotonics Laboratory, CNRS UMR 8250, \\ Paris Descartes University, Sorbonne Paris Cité, 75006 Paris, France \\ ${ }^{2}$ Aix-Marseille University, CNRS, Centrale Marseille, Institut Fresnel UMR 7249, 13013 Marseille, France \\ ${ }^{3}$ Wavefront Engineering Microscopy Group, Neurophotonics Laboratory, CNRS UMR 8250, \\ Paris Descartes University, Sorbonne Paris Cité, 75006 Paris, France
}

(Received 22 July 2016; published 27 January 2017)

\begin{abstract}
Intensity maxima and zeros of speckle patterns obtained behind a diffuser are experimentally interchanged by applying a spiral phase delay of charge \pm 1 to the impinging coherent beam. This transform arises from the expectation that tightly focused beams, which have a planar wave front around the focus, are so changed into vortex beams and vice versa. The statistics of extrema locations and the intensity distribution of the so-generated "complementary" patterns are characterized by numerical simulations. It is demonstrated experimentally that the incoherent superposition of the three "complementary speckle patterns" yield a synthetic speckle grain size enlarged by a factor of $\sqrt{3}$. A cyclic permutation of optical vortices and intensity maxima is unexpectedly observed and discussed.
\end{abstract}

DOI: 10.1103/PhysRevLett.118.043903

The propagation of coherent waves in disordered scattering media is associated with the creation of random wave fields. The resulting inhomogeneous intensity and phase patterns are observed and studied with acoustic waves [1], matter waves [2], and electromagnetic waves [3,4]. Although appearing in random media, the scattering process is deterministic. Thereby, the linear relationship between the incident and scattered wave fields allows controlling the output intensity pattern [5]. However, this approach requires the tedious prior characterization of the scattering properties of the medium for each output mode [6,7]. Angular correlation properties, known as the "memory effect" [8,9], can also be used to retrieve the image of objects hidden by a scattering medium [10-13] but necessitate a thin enough diffuser [14]. Therefore, scattering media are usually considered as a major obstacle for focusing and imaging. Nevertheless, the generated scattered intensity patterns (speckle) feature specific distributions and correlations [15] that can be exploited per se, especially in optical imaging. Uncontrolled speckle patterns used as structured illuminations have been demonstrated to enhance microscopy [16] and photoacoustic imaging [17]. Critical points such as intensity minima also enable subdiffraction tracking of dynamic processes $[18,19]$. Here, we consider capitalizing on both the properties of the medium and the speckle pattern through the unexplored approach: controlling deterministically intrinsic critical points of a random scattered wave field. Full control of the scattered intensity pattern is a difficult task, but predictable relative changes can be straightforward and could modify the spatial location and type of specific speckle features.
Critical points in random scalar wave fields comprise intensity maxima but also zeros of intensity bearing a helical phase structure (vortices). These latter singular points appear with an equal density of topological charges +1 or -1 [20-23]. In free space, a vortex may typically be obtained by imprinting a spiral phase (SP) mask in the Fourier plane of a peaked focused beam [24]. The optical vortex then appears in the coherence area around the initial focus (area of planar wave front). The inverse transform is obtained by applying the SP mask of opposite charge. In speckle patterns, intensity maxima are located in regions of slowly varying phase [25], and the coherence area matches the size of bright speckle spots $[15,26]$. Besides, in the context of telecommunication, it was shown that vortices could be transmitted through scattering media [27-29]. Therefore, it is expected that the intensity maxima and the \pm 1 vortices of a speckle pattern can be interchanged by modulating the wave front impinging onto the disordered scattering medium with a SP of charge $\pm 1\left(\mathrm{SP}^{ \pm 1}\right)$.

The main focus of this Letter is to address in detail this intuitive expectation. We show that intensity maxima and vortices appearing behind a thin scattering medium can actually be exchanged by using a $\mathrm{SP}^{ \pm 1}$. Since intensity maxima and zeros are interchanged, we shall qualify the created speckle patterns as "complementary." In the following, the multiple aspects of this complementarity are experimentally and numerically characterized for coherent optical waves in the light of analytical models. We demonstrate that the location exchange of vortices and intensity maxima also results in complementary spatial arrangements of speckle grains. Finally, two paradoxes raised by vortices-maxima permutations are discussed. 
The typical experimental configuration we use is shown in Fig. 1(a). A laser beam illuminates a diffuser, and spiral phase delays $\mathrm{SP}^{ \pm 1}$ may be imprinted to the wave front with a spatial light modulator (SLM). The SP mask is placed close enough to the diffuser so that the intensity distribution of the impinging beam is minimally altered and all statistical properties of the speckle are preserved. Since energy is also preserved, intensities are normalized by their ensemble average in the following. Complex wave fields and intensities associated with no phase delay and $\mathrm{SP}^{ \pm 1}$ are notated $A_{0}, A_{ \pm 1}$ and $I_{0}, I_{ \pm 1}$, respectively. Maxima of $I_{j}$ and \pm 1 vortices of $A_{j}$ are called $M_{j}$ and $V_{j}^{ \pm}$, respectively [30]. The intuitive expectation is that adding $\mathrm{SP}^{ \pm 1}$ will change intensity maxima $M_{0}$ into zeros $\left(V_{ \pm 1}^{ \pm}\right)$, due to the imprint of a spiral phase on a region of slowly varying phase, and optical vortices $V_{0}^{\mp}$ into intensity maxima $\left(M_{ \pm 1}\right)$, with the opposite transformation. This expectation is supported by considering a simple first-order Taylor expansion of the wave fields under symmetry conditions on the power spectrum [30]. As demonstrated in the context of spiral phase contrast imaging [37,38], an estimate of $A_{ \pm 1}$ in a plane transverse to the propagation axis is given by

$$
A_{ \pm 1}^{(1)}(\mathbf{r})=\mp K \nabla_{\perp} A_{0}(\mathbf{r}) \cdot \sigma_{\mp},
$$

where $\sigma_{+}$and $\sigma_{-}$designate circular vectors $\left[\sigma_{ \pm}=\right.$ $\left(e_{x} \pm i e_{y}\right) / \sqrt{2}$ ] and $K$ is a constant that depends on the power spectrum of $A_{0}$ [30]. The complex wave field amplitude in the vicinity of an intensity maximum $M_{0}$ can be written $A_{0} \propto\left(1-r^{2}\right) e^{i r^{2} \cos (2 \theta)}$, at second-order approximation in $r$ ( $r$ and $\theta$ designating polar coordinates centered on the point of interest). The isotropic amplitude profile $1-r^{2}$ is due to the "congruence" of the intensity maxima [15] and the phase profile to the proximity of the intensity maxima with phase saddles [25] (such a proximity is not observed for nonzero minima [39]). Equation (1) yields $A_{ \pm 1}^{(1)} \propto r e^{ \pm i \theta}$, meaning that $M_{0}$ is changed into a $V_{ \pm 1}^{ \pm}$vortex. Conversely, at a vortex $V_{0}^{ \pm}$, approximating $A_{0}$ by $r e^{ \pm i \theta}$, we obtain $\nabla_{\perp} A_{0} \propto \sigma_{ \pm}$, thus maximizing $I_{1}=\left|A_{1}\right|^{2}$ and minimizing $I_{-1}$ at $V_{0}^{-}$(and vice versa at $V_{0}^{+}$).

An experimental illustration of $V_{0}^{ \pm}$switching to $M_{\mp 1}$ is shown in Figs. 1(b)-1(d). A SLM (LCOS, X10468, Hamamatsu) was used to generate both the scattering phase pattern and phase masks $\mathrm{SP}^{ \pm 1}$. Using the SLM as the diffuser facilitated the phase measurement because a single polarization direction was modulated. The unmodulated polarization component could then serve as a reference beam. The intensity $I_{0}$ [Fig. 1(b)] and the phase of $A_{0}$ [Fig. 1(c)] were measured by phase-stepping interferometry illuminating the parallel-aligned liquid crystal SLM with a $45^{\circ}$ polarized beam. $V_{0}^{ \pm}$deduced from the phase map are superposed with $I_{1}$ and $I_{-1}$ [Fig. 1(d)] and validate the high correlation with $M_{\mp 1}$.

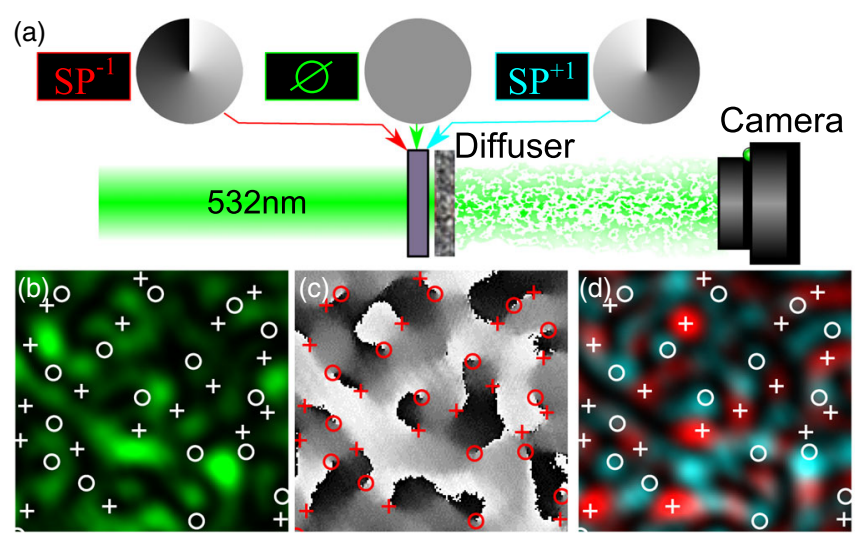

FIG. 1. Experimental generation of complementary speckle patterns. (a) Spiral phase delays of charge -1 and $+1\left(\mathrm{SP}^{\mp 1}\right)$ are introduced by a spatial light modulator on the beam impinging onto the diffuser. Speckle patterns corresponding to each configuration are recorded on a camera. The intensity (b) and phase (c) patterns of $A_{0}$ were measured experimentally by phase-stepping digital holography. +1 and -1 vortices of $A_{0}$ are identified with + and $\circ$ symbols, respectively. Intensity patterns $I_{1}$ (cyan) and $I_{-1}$ (red) resulting from the addition of $\mathrm{SP}^{ \pm 1}$ are also compared to vortices of $A_{0}(\mathrm{~d})$. The maxima of $I_{1}$ and $I_{-1}$ exhibit a high correlation with vortices of $A_{0}$.

To quantify the exchange between $M_{0}$ and $V_{1}^{+}$, we computed the radial probability density function (RPDF) of the distance between $V_{1}^{+}$and the closest $M_{0}$ [notated $\left.d\left(V_{1}^{+}, M_{0}\right)\right]$ [Fig. 2(a)]. This $\operatorname{RPDF}(r)$ corresponds to the probability to find the closest $M_{0}$ at the distance $r$ from a vortex $V_{1}^{+}$per unit area. Statistical distributions were assessed from numerical simulations [30] of Gaussian random wave fields in a transverse plane, to process large numbers of critical points in a well-controlled configuration. Distances are normalized to $\lambda /(2 \mathrm{NA}$ ) (where $\lambda$ is the wavelength and NA the numerical aperture of illumination), i.e., the full width at half maximum (FWHM) of a speckle grain. The RPDF of $d\left(V_{0}^{-}, M_{1}\right)$ is the same as for $d\left(V_{1}^{+}, M_{0}\right)$ due to the symmetry of the SP transform [Fig. 2(a)]. For comparison, the RPDF for $d\left(V_{0}^{+}, M_{0}\right)$ is plotted. The RPDF
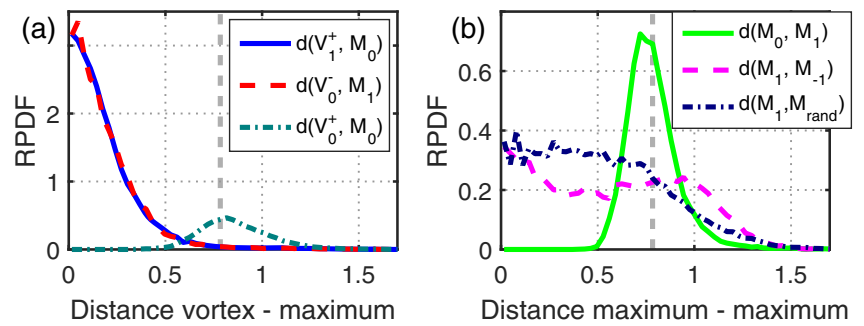

FIG. 2. RPDFs of separation distances between one set of critical points and the closest points of another set, obtained from numerical simulations. The abscissa unit is $\lambda /(2 \mathrm{NA})$. The distance between $V_{1}^{+}$and the closest $M_{0}$ is notated $d\left(V_{1}^{+}, M_{0}\right)$. Vertical thick dashed gray lines correspond to the correlation length $l_{c} \approx 0.78$. 
of $d\left(V_{1}^{+}, M_{0}\right)$ is tightly confined at zero distances, confirming the interchange induced by the SP mask, while maxima $M_{0}$ and vortices $V_{0}$ in a speckle pattern are statistically separated by a larger average distance of about one correlation length $l_{c} \approx 0.78$ [15]. The RPDF of the distance between $M_{0}$ and the closest $M_{ \pm 1}$ (correlating with $V_{0}^{\mp}$ ) exhibits an exclusion distance equal to this correlation length [Fig. 2(b)]. The peak of this latter RPDF is centered at $l_{c}$, which is also the radius of the donut that would be obtained by adding $\mathrm{SP}^{ \pm 1}$ to the wave front of a focused beam propagating in free space. Therefore, bright speckle spots (around the maxima) of $I_{1}$ and $I_{-1}$ will be minimally overlapping with those of $I_{0}$, which is verified experimentally [Fig. 3(a)]. For comparison, the RPDFs of the distance between $M_{1}$ and $M_{-1}$ as well as the distance between $M_{1}$ and the maxima of a noncorrelated speckle pattern are also shown in Fig. 2(b). Although no exclusion distance is observed between $M_{1}$ and $M_{-1}$, the lower RPDF of $d\left(M_{1}, M_{-1}\right)$ for small distances indicates a partial repulsion of bright speckle spots of $I_{1}$ and $I_{-1}$. The repulsions between the maxima of the complementary speckle patterns reveal a new facet of the complementarity in terms of the spatial arrangement of speckle grains.

Experimentally, the complementarity of spatial arrangements of bright speckle spots was first demonstrated using a surface scatterer, preserving the incident polarization state of the beam (120 grit ground glass diffuser, Thorlabs). Overlaying $I_{0}, I_{1}$, and $I_{-1}$, speckle grains are observed to be closely packed and to create a dense mapping of the plane [Fig. 3(a)]. The characteristic length scale of the spatial fluctuation of the sum of $I_{0}+I_{1}$ is therefore observed to be larger than for a single speckle pattern [Fig. 3(b)]. The $\sqrt{2}$ factor on the FWHM of the autocorrelation function (ACF) corresponds to a doubling of the coherence area, since speckle grains of $I_{0}$ and $I_{1}$ minimally overlap. Similarly, the ACF FWHM of the sum $I_{0}+I_{1}+I_{-1}$ is increased by a factor of $\sqrt{3}$ [Fig. 3(b)], corresponding to a tripling of the coherence area, since $M_{1}$

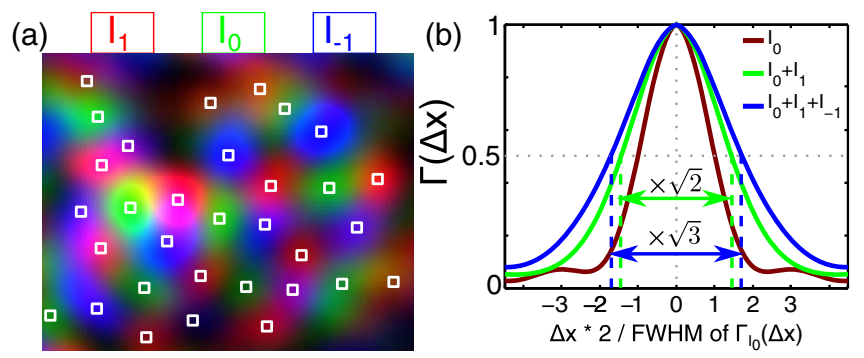

FIG. 3. Speckle patterns $I_{0}, I_{1}$, and $I_{-1}$ measured through a ground glass diffuser and overlapped, coded in saturated red green blue colors (a). The locations of the maxima are marked with squares, illustrating a dense mapping of the plane. Cross sections of the (normalized) autocorrelation functions of $I_{0}$, the sum $I_{0}+I_{1}$, and the sum $I_{0}+I_{1}+I_{-1}$ demonstrate the complementarity of speckle patterns (b). and $M_{-1}$ partially repulse. In contrast, no enlargement of the ACF is observed for the sum of uncorrelated speckle patterns. Consequently, the induced speckle complementarity allows the synthetic enlargement of speckle grains and thus provides the possibility to uniquely build up low spatial frequencies of the intensity distribution by incoherent summation. Interestingly, the first-order statistics of the sum $I_{0}+I_{1}+I_{-1}$ were verified to match those of the sum of three random speckle patterns [26], meaning that the complementarity holds only on a local scale. To go beyond surface scattering, we conducted an experiment through a $0.7-\mathrm{mm}$-thick slice of chicken breast, sandwiched between two glass slides. This thickness corresponds to several mean-free paths $(\sim 30 \mu \mathrm{m}[40])$ and is even of the order of the tissue transport mean-free path [41]. The sample also depolarizes the incident laser beam. Decorrelation was negligible during the sequential recording of patterns. In this experimental configuration, $I_{0}+I_{1}+I_{-1}$ was found to yield the same enlargement of the ACF as for the surface diffuser [30]. Despite depolarization behind the sample, the complementarity was observed for each transverse polarization component and is also expected to be verified for the longitudinal component of the field [30]. The deterministic generation of complementary speckle patterns is then robust through several scattering mean free path. We interpret that complementary speckle generation requires a minimalistic "memory effect" [8,9] over a distance as short as a single correlation length. For thicker samples (thickness $\sim 1.4 \mathrm{~mm}$ ), the complementarity was observed to degrade $(\sim 1.2$ increase in the ACF FWHM instead of $\sqrt{3})$.

In addition to spatial correlations between vortices and intensity maxima, intensity values $I_{1}$ and $I_{-1}$ are expected to exhibit particular statistical properties at the locations of $V_{0}^{ \pm}$. We derive here an analytical treatment of this statistical behavior and validate it by a numerical simulation. For a fully developed speckle pattern exhibiting Gaussian statistics and with a power spectrum having circular symmetry, it can be shown that the joint probability density function (PDF) of complementary speckle patterns is [26]

$$
\rho\left(I_{0}, I_{1}^{(1)}, I_{-1}^{(1)}\right)=e^{-I_{0}} \times \alpha^{2} e^{-\alpha\left(I_{1}^{(1)}+I_{-1}^{(1)}\right)},
$$

where $I_{ \pm 1}^{(1)}$ are defined according to Eq. (1) and $\alpha=\left\langle I_{ \pm 1}^{(1)}\right\rangle^{-1}$ slightly differs from $\left\langle I_{0}\right\rangle=1$ due to the first-order approximation. The PDF of intensities $I_{ \pm 1}$ at locations of $V_{0}^{ \pm}$is calculated by integrating Eq. (2), using the property that, at first order, the charge of the optical vortices associated with zeros of $I_{0}$ is given [30] by the sign of $I_{-1}^{(1)}-I_{1}^{(1)}$ as confirmed in Fig. 4(a) (and validated numerically for $93 \%$ of vortices). Noteworthy, nonzero stationary points of $I_{0}$ lie on nodal lines $I_{-1}^{(1)}=I_{1}^{(1)}$ [30].

The conditional PDF of $I_{1}^{(1)}$ at $V_{0}^{+}$and $V_{0}^{-}$can be calculated [30]: 

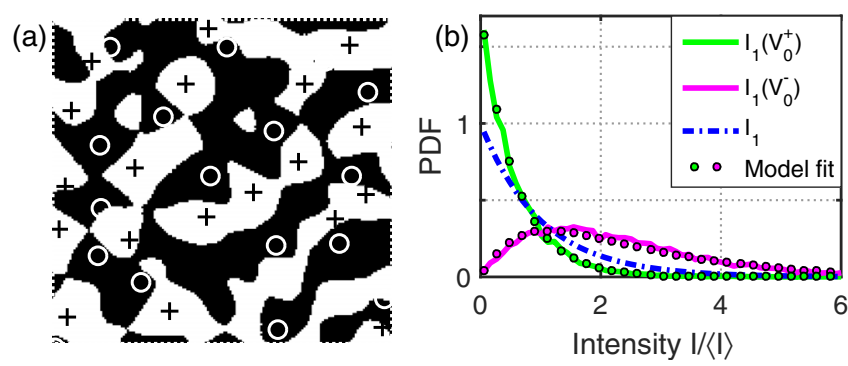

FIG. 4. From experimental data shown in Fig. 1, the sign of $I_{-1}-I_{1}$, represented in black (negative) and white (positive), identifies the sign of vortices $V_{0}^{ \pm}$(a). PDFs of intensities $I_{1}$ at optical vortices of $A_{0}$ (b) deduced from numerical simulations (solid lines) and from fits of first-order approximation functions. The PDFs are compared with the PDF of speckle patterns $I_{1}$.

$$
\begin{gathered}
\rho_{V_{0}^{-}}\left(I_{1}^{(1)}\right)=2 \alpha e^{-\alpha I_{1}^{(1)}}\left(1-e^{-\alpha I_{1}^{(1)}}\right), \\
\rho_{V_{0}^{+}}\left(I_{1}^{(1)}\right)=2 \alpha e^{-2 \alpha I_{1}^{(1)}} .
\end{gathered}
$$

Equations (3) and (4) are plotted in Fig. 4(b) and were found to match PDFs from numerical simulations with fitting coefficients $\alpha=0.60$ for Eq. (3) and $\alpha=0.86$ for Eq. (4). The difference in the value of $\alpha$ for +1 and -1 vortices of $A_{0}$ is attributed here to the limit of the first-order approximation. In addition, $V_{0}^{+}$and $V_{0}^{-}$experience an asymmetric transformation in $I_{1} . V_{0}^{-}$yield the maxima of $I_{1}$, and the intensity at these points is statistically larger than the average intensity: $\left\langle I_{1}^{(1)}\left(V_{0}^{-}\right)\right\rangle=3 /(2 \alpha)$. In contrast, $V_{0}^{+}$cannot result in +2 -charged optical vortices of $A_{1}$, since such structures are unstable and do not appear in Gaussian random wave fields [42]. Vortex charges of the same sign cannot be simply added, but still $V_{0}^{+}$yield $I_{1}$ values statistically lower than the average intensity: $\left\langle I_{-1}^{(1)}\left(V_{0}^{-}\right)\right\rangle=1 /(2 \alpha)$. The first-order model is found to nicely account for the PDFs with an adjustment of a single coefficient.

Integration of Eq. (2) at $M_{0}$ cannot easily result in an analytical expression [43]. From numerical simulations, the PDF of intensities $I_{1}$ at $M_{0}$ was found to have a negative exponential distribution with a rate parameter of 2.4. Therefore, although $M_{0}$ do not coincide exactly with $V_{1}^{ \pm}$ vortices, the intensity at these points, after adding $\mathrm{SP}^{ \pm 1}$, is smaller than the average intensity. The rate parameter is nevertheless influenced by the discrepancy between the number of $M_{0}$ and the number of $V_{1}^{+}$and weighted by maxima that do not turn into vortices. In conclusion, deterministic intensity fluctuations can be induced locally with $\mathrm{SP}^{ \pm 1}$ masks. On a larger scale, intensity fluctuations across the complementary speckle patterns enhance low spatial frequencies [30].

Although demonstrating the complementarity of the sogenerated speckle patterns, the intuitive interpretation of

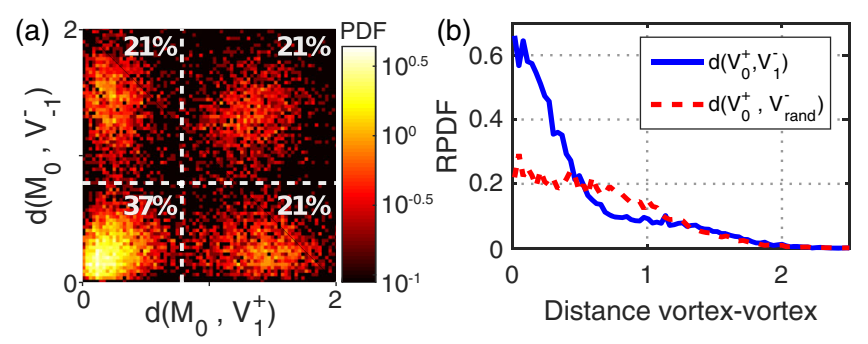

FIG. 5. Joint PDF of distances $d\left(M_{0}, V_{1}^{+}\right)$and $d\left(M_{0}, V_{1}^{-}\right)$in the logarithmic color scale (a) illustrating that some maxima are not changed into vortices. The fraction of maxima populations is indicated in each quadrant. The RPDF of the distance between vortices $d\left(V_{0}^{+}, V_{1}^{-}\right)$showing a correlation of the locations of these two populations of vortices (b).

the $\mathrm{SP}^{ \pm 1}$ transform fails to interpret two main fundamental paradoxes. The first paradox arises from the different density of vortices [21,22] and intensity maxima [43] in Gaussian random wave fields. The larger density of maxima makes an one-to-one exchange impossible. In Fig. 5(a), the joint PDF of distances $d\left(M_{0}, V_{1}^{+}\right)$and $d\left(M_{0}, V_{-1}^{-}\right)$obtained from a numerical simulation is plotted for a ratio between the number of $M_{0}$ and the number of $V_{1}^{+}$of $\sim 1.6$. The joint PDF exhibits two bifurcation lines at a distance of $l_{c}$, separating four populations of maxima $M_{0}$. Thus, $M_{0}$ is interpreted to turn into a vortex of the same charge as the SP mask only when the distance to the vortex is shorter than $l_{c}$. When a maximum $M_{0}$ is not changed into a vortex, the maximum distance probability for the nearest vortex is cast at $2 \times l_{c}$. Moreover, despite vortices being less numerous, for $8 \%$ of $V_{0}^{-}$the closest $M_{1}$ is located beyond $l_{c}$ [Fig. 2(a)], and thus such vortices are not transformed into maxima through a $\mathrm{SP}^{+1}$ mask. The second paradox arises from the question of the zero average of the topological charge [15]. If the topological charge of all maxima and vortices is incremented by adding a $\mathrm{SP}^{+1}$ mask, then the total charge of $A_{1}$ would diverge as their number. Moreover, $V_{0}^{+}$cannot possibly result in +2 vortices [42]. Since we obtained from numerical simulations that $92 \%$ of $V_{0}^{-}$are changed into $M_{1}$ and that $92 \%$ of $V_{1}^{+}$arise from $M_{0}$ given a $l_{c}$ distance threshold, we must conclude that, in a cyclic permutation logic, most of $V_{0}^{+}$are unexpectedly responsible for the creation of vortices $V_{1}^{-}$. We thus studied numerically the correlation in the location of $V_{0}^{+}$and $V_{1}^{-}$. In comparison with two random distributions of vortices, the RPDF of distances $d\left(V_{0}^{+}, V_{1}^{-}\right)$indeed exhibits a peak at small distances [Fig. 5(b)], demonstrating a significant correlation between the locations of $V_{0}^{+}$and $V_{1}^{-}$. The two paradoxes raise exciting fundamental questions on the rules guiding the interchange of vortices and maxima and the quantification of the distances in the frame of random wave fields and their multiple correlations [15]. They would deserve further investigation in future studies. 
In conclusion, we demonstrated the possibility to generate complementary speckle patterns through surface and thick diffusers by modulating the impinging wave front with spiral phases. The complementarity was characterized in terms of maximum-vortex interchange and deterministic intensity fluctuation at these particular points, as well as in terms of closely packed bright speckle grains. The sum and the fluctuation of the complementary intensity patterns, on a per pixel basis, were found to synthetically enhance the low spatial frequency with as few as two speckle patterns. The limits of the one-to-one transformation of intensity extrema was discussed as well as the possibility of a cyclic permutation logic, introducing fundamental questions on the process. In spite of its fundamental complexity, the proposed complementary speckle generation bears the advantage of being easy to implement experimentally and is expected to work as long as the spiral wave front is transmitted through the diffuser.

The authors acknowledge Robert Kuszelewicz, Joseph Zyss, and Valentina Emiliani for stimulating discussions. This work was supported by grants from the Région Ile-deFrance, the French-Israeli Laboratory $\mathrm{NaBi}$, and the Centre National de la Recherche Scientifique.

* Corresponding author. marc.guillon@parisdescartes.fr

[1] R. F. Wagner, S. W. Smith, J. M. Sandrik, and H. Lopez, IEEE Trans. Sonics Ultrason. 30, 156 (1983).

[2] R. G. Dall, S. S. Hodgman, A. G. Manning, M. T. Johnsson, K. G. H. Baldwin, and A. G. Truscott, Nat. Commun. 2, 291 (2011).

[3] J. Wang and A. Z. Genack, Nature (London) 471, 345 (2011).

[4] M. Sutton, S. G. J. Mochrie, T. Greytak, S. E. Nagler, L. E. Berman, G. A. Held, and G. B. Stephenson, Nature (London) 352, 608 (1991).

[5] A. P. Mosk, A. Lagendijk, G. Lerosey, and M. Fink, Nat. Photonics 6, 283 (2012).

[6] S. M. Popoff, G. Lerosey, R. Carminati, M. Fink, A. C. Boccara, and S. Gigan, Phys. Rev. Lett. 104, 100601 (2010).

[7] R. Horstmeyer, H. Ruan, and C. Yang, Nat. Photonics 9, 563 (2015).

[8] I. Freund, M. Rosenbluh, and S. Feng, Phys. Rev. Lett. 61, 2328 (1988).

[9] S. Feng, C. Kane, P. A. Lee, and A. D. Stone, Phys. Rev. Lett. 61, 834 (1988).

[10] A. Labeyrie, Astron. Astrophys. 6, 85 (1970).

[11] O. Katz, P. Heidmann, M. Fink, and S. Gigan, Nat. Photonics 8, 784 (2014).

[12] J. Bertolotti, E. G. van Putten, C. Blum, A. Lagendijk, W. L. Vos, and A. P. Mosk, Nature (London) 491, 232 (2012).

[13] X. Yang, Y. Pu, and D. Psaltis, Opt. Express 22, 3405 (2014).
[14] S. Schott, J. Bertolotti, J.-F. Léger, L. Bourdieu, and S. Gigan, Opt. Express 23, 13505 (2015).

[15] I. Freund, Waves Random Media 8, 119 (1998).

[16] E. Mudry, K. Belkebir, J. Girard, J. Savatier, E. L. Moal, C. Nicoletti, M. Allain, and A. Sentenac, Nat. Photonics 6, 312 (2012).

[17] J. Gateau, T. Chaigne, O. Katz, S. Gigan, and E. Bossy, Opt. Lett. 38, 5188 (2013).

[18] W. Wang, T. Yokozeki, R. Ishijima, A. Wada, Y. Miyamoto, M. Takeda, and S. G. Hanson, Opt. Express 14, 120 (2006).

[19] S. J. Kirkpatrick, K. Khaksari, D. Thomas, and D. D. Duncan, J. Biomed. Opt. 17, 050504 (2012).

[20] J. Nye and M. Berry, Proc. R. Soc. A 336, 165 (1974).

[21] M. V. Berry, J. Phys. A 11, 27 (1978).

[22] N. Baranova, B. Zel'dovich, A. Mamaev, N. Pilipetskii, and V. Shkukov, JETP Lett. 33, 195 (1981).

[23] I. Freund, Phys. Lett. A 198, 139 (1995).

[24] M. R. Dennis, K. O'Holleran, and M. J. Padgett, Progr. Optic. 53, 293 (2009).

[25] N. Shvartsman and I. Freund, Opt. Commun. 117, 228 (1995).

[26] J. W. Goodman, Speckle Phenomena in Optics (Roberts and Co., Englewood, CO, 2006).

[27] W. B. Wang, R. Gozali, L. Shi, L. Lindwasser, and R. R. Alfano, Opt. Lett. 41, 2069 (2016).

[28] G. R. Salla, C. Perumangattu, S. Prabhakar, A. Anwar, and R. P. Singh, Appl. Phys. Lett. 107, 021104 (2015).

[29] G. Gbur and R. K. Tyson, J. Opt. Soc. Am. A 25, 225 (2008).

[30] See Supplemental Material at http://link.aps.org/ supplemental/10.1103/PhysRevLett.118.043903 for notations, detailed calculations, precisions about numerical simulations and experiments as well as additional numerical and experimental data, which includes Refs. [26,31-36].

[31] J. Masajada and B. Dubik, Opt. Commun. 198, 21 (2001).

[32] S. Vyas and P. Senthilkumaran, Appl. Opt. 46, 2893 (2007).

[33] Z. Li and C. Cheng, Appl. Opt. 53, 1629 (2014).

[34] S.-C. Pei and C.-L. Liu, in Proceedings of the IEEE International Conference on Acoustics, Speech and Signal Processing (ICASSP), Kyoto, 2012 (IEEE, New York, 2012), pp. 3701-3704.

[35] M. Berry and M. Dennis, Proc. R. Soc. A 456, 2059 (2000).

[36] B. Richards and E. Wolf, Proc. R. Soc. A 253, 358 (1959).

[37] A. Jesacher, S. Fürhapter, S. Bernet, and M. Ritsch-Marte, J. Opt. Soc. Am. A 23, 1400 (2006).

[38] M. Guillon and M. Lauterbach, J. Opt. Soc. Am. A 31, 1215 (2014).

[39] I. Freund and N. Shvartsman, Phys. Rev. E 51, 3770 (1995).

[40] Z. Yaqoob, D. Psaltis, M. S. Feld, and C. Yang, Nat. Photonics 2, 110 (2008).

[41] V. Ntziachristos, Nat. Methods 7, 603 (2010).

[42] I. Freund, N. Shvartsman, and V. Freilikher, Opt. Commun. 101, 247 (1993).

[43] A. Weinrib and B. I. Halperin, Phys. Rev. B 26, 1362 (1982). 